\title{
Spinal Cord Compression Secondary to a Spontaneous Spinal Haematoma in a Patient Newly Treated with Rivaroxaban
}

\author{
Javier Guerrero-Niño ${ }^{1}$, Sara De Cesaris ${ }^{1}$, Xavier Jannot ${ }^{2}$, Noel Lorenzo-Villalba $^{2}$ \\ ${ }^{1}$ Service des Urgences, Hôpitaux Universitaires de Strasbourg, Strasbourg, France \\ ${ }^{2}$ Service de Médecine Interne, Diabète et Maladies Métaboliques, Hôpitaux Universitaires de Strasbourg, Strasbourg, France
}

Received: $26 / 04 / 2021$

Accepted: 02/05/2021

Published: 10/05/2021

How to cite this article: Guerrero-Niño J, De Cesaris S, Jannot X, Lorenzo-Villalba N. Spinal cord compression secondary to a spontaneous spinal haematoma in a patient newly treated with rivaroxaban. EJCRIM 2021;8: doi:10.12890/2021_002593.

Conflicts of Interests: The authors declare there are no competing interests.

This article is licensed under a Commons Attribution Non-Commercial 4.0 License

\section{ABSTRACT}

A 74-year-old patient anticoagulated with rivaroxaban for chronic atrial fibrillation presented to the emergency department with acute lumbar pain with progressive weakness of the lower limbs and inability to stand up. No previous trauma was reported. Neurological examination was consistent with a complete spinal cord syndrome at the level of T6. Magnetic resonance imaging showed the presence of spinal cord compression associated with signs of extensive intramedullary inflammation secondary to a haematoma. The patient underwent thoracic laminectomy with evacuation of an intradural haematoma. No intraoperative complications were described, but no clinical improvement had been achieved 15 days after the surgical intervention.

\section{LEARNING POINTS}

- Apixaban could be preferred as the first choice for direct oral anticoagulation (DOAC) in older patients.

- DOACs can have important side effects even if the switch from vitamin K antagonist to this group is correctly carried out.

\section{KEYWORDS}

Rivaroxaban, major bleeding, complete flaccid paraplegia, anticoagulation

\section{INTRODUCTION}

Direct oral anticoagulants (DOACs) are currently the first-line treatment in two clinical conditions: the prevention of stroke and systemic embolism in patients with non-valvular atrial fibrillation (NVAF), and the treatment/prevention of venous thromboembolism ${ }^{[1]}$. DOAC dosage is based on evaluation of the clinical indication, age, sex, body weight, concomitant administration of other drugs, and renal and liver function. Bleeding is the most frequent complication of anticoagulant therapy, while other complications such as allergic reactions, thrombocytopenia, skin necrosis, purple toes or alopecia are rare ${ }^{[2]}$. Major bleeding complications may also occur in patients treated with DOACs, accounting for 3-4\% of bleeds per patient-year ${ }^{[3]}$.

\section{CASE DESCRIPTION}

A 74-year-old patient, anti-coagulated with rivaroxaban for chronic atrial fibrillation, presented during the night at about 4:00 a.m. to the emergency department complaining of acute lumbar pain with progressive weakness of the lower limbs and inability to stand up.

The patient had no history of fever, chills or weight loss but reported progressive shortness of breath during the 48 hours prior to hospital admission. 
Her past medical history was significant for chronic atrial fibrillation previously treated with vitamin $\mathrm{K}$ antagonist and switched to rivaroxaban $20 \mathrm{mg}$ daily 5 days before hospital admission, atorvastatin $10 \mathrm{mg}$ daily, furosemide $40 \mathrm{mg}$ daily, spironolactone $25 \mathrm{mg}$ daily and bisoprolol $5 \mathrm{mg}$ twice daily. There were no newly introduced medications. Her family history was non-contributory. She had no known drug or food allergies, as well as no history of smoking, alcohol abuse or illicit drug use. No recent trauma or exertion was reported.

On physical examination, her heart rate was 95 beats/min, blood pressure was 148/102 mmHg, respiratory rate was 15 breaths/min, and oxygen saturation was $98 \%$ on room air. The patient was alert and oriented to time, place and person. A complete spinal cord syndrome at level T6 was found. Dorsolumbar spinal pain centred around the thoracolumbar hinge, with no sensory-motor deficit of the upper extremities, complete flaccid paraplegia (0/5), T6 territory hypoesthesia with tuning fork apallesthesia extending to the sternum, and anal atony with hollowness and presence of stool at rectal touch. Reflexes were not perceived in the lower limbs, The Babinski sign was present, and no epileptoid tremor was found. Heart sounds were irregular without any rubs or murmurs. The lungs were clear. The abdomen was tender and non-distended, without masses.

Blood tests showed leucocytosis $(15.5 \mathrm{~g} / \mathrm{l})$ with predominance of polynuclear neutrophils $(14.2 \mathrm{~g} / \mathrm{l})$ and with normal platelet count and C-reactive protein $(9.3 \mathrm{mg} / \mathrm{l}$ ). Haemoglobin was $12.8 \mathrm{~g} / \mathrm{dl}$ and mean corpuscular volume was $102 \mathrm{fl}$. Prothrombin time (PT) was $62 \%$, activated partial thromboplastin time (APTT) was 30.1 (21-35 seconds), INR 1.42, fibrinogen $3.37 \mathrm{~g} / \mathrm{l}$, and anti-factor X activity $118.89 \mathrm{ng} / \mathrm{ml}$. Liver function tests showed increased total bilirubin (27 $\mu \mathrm{mol} / \mathrm{I})$, levels of alanine transaminase (ALT; 166 IU/I), aspartate transaminase (AST; 179 $\mathrm{IU} / \mathrm{I}$ ) and alkaline phosphatase (171 IU/I), while gamma-glutamyl transferase was normal. The glomerular filtration rate (GFR) was $69 \mathrm{ml} / \mathrm{min}$, serum creatinine $73 \mu \mathrm{mol} / \mathrm{l}$, serum sodium $133 \mathrm{mmol} / \mathrm{l}$ and potassium $5.2 \mathrm{mmol} / \mathrm{I}$. The rest of the electrolyte and thyroid function tests were within the normal range. The electrocardiogram showed atrial fibrillation with controlled ventricular response.

Magnetic resonance imaging showed a left posterolateral intracanal collection of probably epidural topography at the level of the T4-T5-T6 vertebrae that extended over $54 \mathrm{~mm}$ and measured $12 \mathrm{~mm}$ in the anteroposterior axis. It showed a discreetly heterogeneous signal in T2 and STIR hypersignal, without clear translation on the T1 sequence, and without enhancement after injection. A 4 mm pumiceous contrast image was noted opposite T6, with a linear contrast image above it that appeared to be vascular. On the axial T2-Medic sequence, hyposignals in favour of hemosiderin deposits were noted. The lesion exerted a mass effect on the medullary cord, which was displaced to the right, the site of an intramedullary STIR hypersignal extending from T3 to T7. The height of the vertebral bodies was preserved without vertebral fracture. There was no signal abnormality of the terminal cone (Fig. 1).
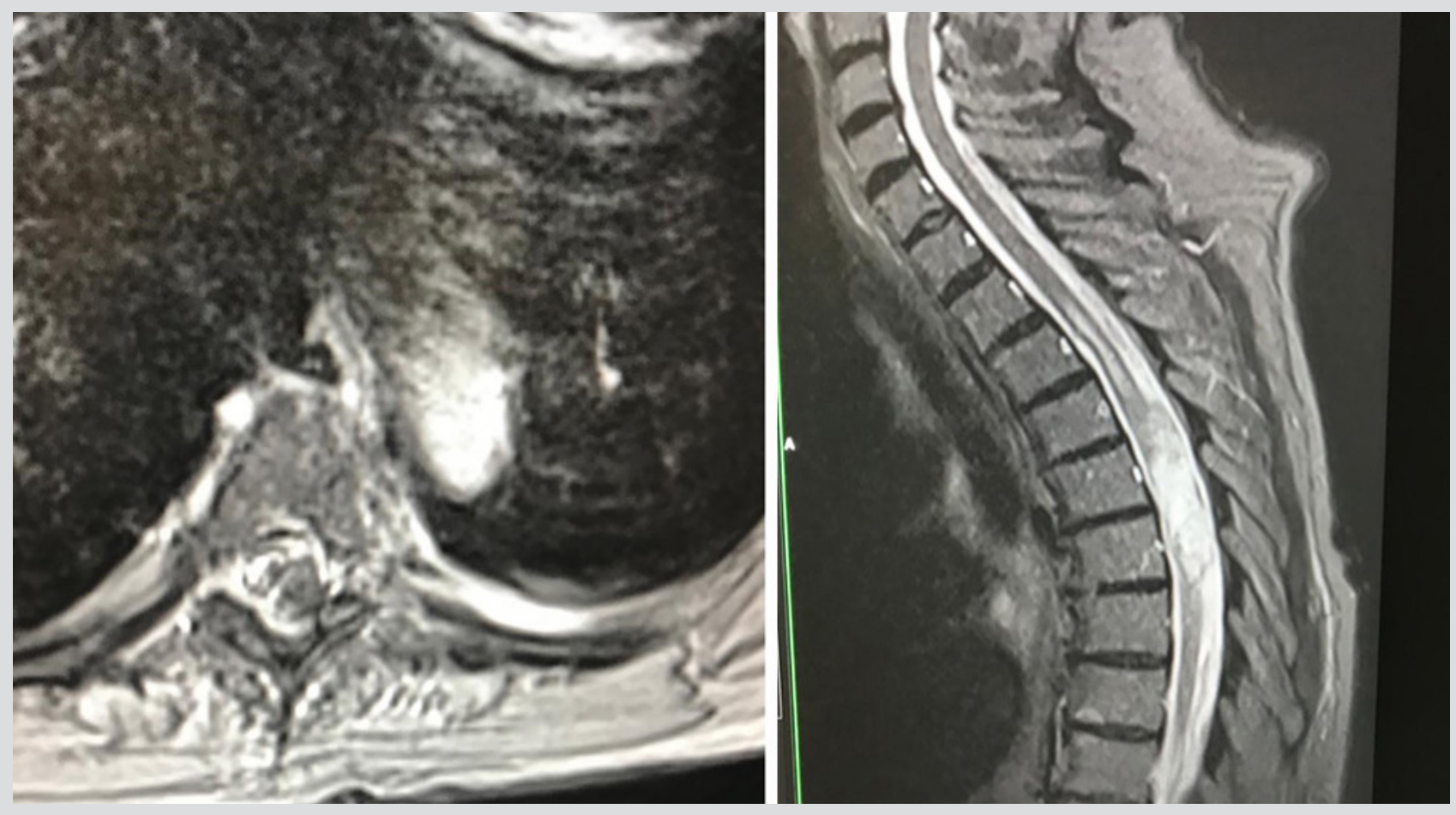

Figure 1. Magnetic resonance imaging findings 
Human prothrombin complex $4000 \mathrm{IU}$ was administered in the emergency department. Neurosurgeons performed a laminectomy centred on the affected thoracic vertebral levels with evacuation of an intradural haematoma at level T4-T5-T6. Haemostasis was difficult throughout the procedure but no intra- or extradural bleeding was reported at the end of the procedure.

Fifteen days after the procedure, no neurological improvement was noted.

\section{DISCUSSION}

Spontaneous spinal haematomas are rare and complex spinal pathologies leading to devastating neurological outcomes. They can be idiopathic or arise as the result of rupture of the epidural venous plexus, vascular abnormalities, arteriovenous malformation, vertebral haemangiomas, lumbar puncture, spinal manipulation, coagulopathies, or pharmacological anticoagulation ${ }^{[4]}$.

Anticoagulant drugs are prescribed to treat and prevent thrombotic events. Drug levels are important as low drug levels could be insufficient to prevent coagulation activation and thrombus formation, while high drug levels may favour or cause bleeding complications. It has been reported that some patients may have a considerable risk of bleeding that is independent of anticoagulation because of various conditions such as age, comorbidities, comedications and impaired renal function. High interindividual variability in drug plasma levels has been shown with all DOACs, and an association between plasma levels and major events has been highlighted by FDA reports on DOAC phase III clinical studies $^{[1,5]}$.

Rivaroxaban was prescribed in the presented case 5 days prior to hospital admission. During hospitalization, we confirmed that the anticoagulation treatment was correctly switched from vitamin $\mathrm{K}$ antagonist to rivaroxaban. In our case, rivaroxaban was imputed as the causative drug leading to the development of the blood collection. It has been recommended that rivaroxaban should be started after warfarin has been stopped and INR is at or below 2.5. The Rivaroxaban versus Warfarin in Nonvalvular Atrial Fibrillation (ROCKET-AF) trial (comparing rivaroxaban to warfarin for the prevention of stroke and systemic embolic events (SEE) in NVAF) found that patients with a prior history of gastrointestinal (GI) bleeding were at increased risk of major bleeding with rivaroxaban as compared with warfarin ${ }^{[6]}$. Rivaroxaban caused a significantly higher risk of haemoglobin decrease $\geq 2 \mathrm{~g} / \mathrm{dl}$ and transfusion compared with warfarin, but critical bleeding and fatal bleeding were significantly lower in rivaroxaban-treated patients.

In this study, major bleeding in patients treated with rivaroxaban was represented in order of importance by gastrointestinal, intracranial, intraparenchymal, intraventricular, subdural and subarachnoid haematoma, but no epidural haematoma was reported. In contrast to warfarin, there is no specific antidote for neutralizing the activity of DOAC ${ }^{[7]}$, which makes treatment of major bleeding challenging.

Medical history and comorbidities may drive the choice of a particular DOAC. Apixaban has been suggested as a reasonable first choice either in older patients or in subjects with chronic renal failure ${ }^{[8]}$. The recently updated 2019 American Geriatrics Society Beers criteria recommend a cautious use of dabigatran and rivaroxaban in patients with AF aged $\geq 75$ years ${ }^{[9]}$.

\section{CONCLUSIONS}

Major bleeding complications may arise in elderly patients treated with DOAC. The DOAC should be carefully chosen following the current clinical recommendations.

\section{REFERENCES}

1. Testa S, Legnani C, Antonucci E, Paoletti O, Dellanoce C, Cosmi B, et al; Coordinator of START2-Register. Drug levels and bleeding complications in atrial fibrillation patients treated with direct oral anticoagulants. J Thromb Haemost 2019;17(7):1064-1072.

2. Schulman S, Kearon C; Subcommittee on Control of Anticoagulation of the Scientific and Standardization Committee of the International Society on Thrombosis and Haemostasis. Definition of major bleeding in clinical investigations of antihemostatic medicinal products in non-surgical patients. J Thromb Haemost 2005;3(4):692-694.

3. Beyer Westendorf J, Forster K, Pannach S, Ebertz F, Gelbricht V, Thieme C, et al. Rates, management, and outcome of rivaroxaban bleeding in daily care: results from the Dresden NOAC registry. Blood 2014;124:955-962.

4. Fiani B, Jarrah R, Fiani NJ, Runnels J. Spontaneous cervical epidural hematoma: insight into this occurrence with case examples. Surg Neurol Int 2021;12:79.

5. Nguyen SN, Ruegger MC, Salazar E, Dreucean D, Tatara AW, Donahue KR. Evaluation of anti-Xa apixaban and rivaroxaban levels with respect to known doses in relation to major bleeding events. J Pharm Pract 2021 Apr 12:8971900211009075.

6. Goodman SG, Wojdyla DM, Piccini JP, et al. Factors associated with major bleeding events: insights from the ROCKET AF trial (rivaroxaban once-daily oral direct factor Xa inhibition compared with vitamin K antagonism for prevention of stroke and embolism trial in atrial fibrillation). J Am Coll Cardiol 2014;63(9):891-900.

7. Massicotte A. A practice tool for the new oral anticoagulants [published correction appears in Can Pharm J (Ott). 2014;147(5):319]. Can Pharm J (Ott) 2014;147(1):25-32.

8. Diener H-C, Aisenberg J, Ansell J, Atar D, Breithardt G, Eikelboom J, et al. Choosing a particular oral anticoagulant and dose for stroke prevention in individual patients with non-valvular atrial fibrillation: part 1. Eur Heart J 2017;38(12):852-859.

9. By the 2019 American Geriatrics Society Beers Criteria ${ }^{\circledR}$ Update Expert Panel. American Geriatrics Society 2019 Updated AGS Beers Criteria ${ }^{\circledR}$ for Potentially Inappropriate Medication Use in Older Adults. J Am Geriatr Soc 2019;67(4):674-694. 